\title{
METHODS TO ASSESS THE STABILITY OF A BICYCLE RIDER SYSTEM
}

\author{
Adrian Cooke, \\ Marc Beusenberg, Maarten Bonnema, \\ Wim Poelman \\ Design Production and Engineering \\ Engineering Technology \\ University of Twente \\ Enschede, Overijssel, 7522 NB \\ The Netherlands, \\ Email: Adrian.Cooke@utwente.nl, \\ C.M.Beusenberg@utwente.nl, \\ G.M.Bonnema@utwente.nl, \\ W.A.Poelman@utwente.nl
}

\author{
Vera Bulsink, \\ Bart Koopman \\ Bio-mechanical engineering \\ Engineering Technology \\ University of Twente \\ Enschede, Overijssel, 7522 NB \\ The Netherlands, \\ Email: Vera.Bulsink@utwente.nl \\ H.F.J.M.Koopman@utwente.nl
}

\author{
Rosemary Dubbeldam, \\ Roessingh Research and Development, \\ Roessinghsbleekweg 33b \\ Enschede, Overijssel, $7522 \mathrm{AH}$ \\ The Netherlands \\ Email: R.Dubbeldam@rrd.nl
}

\section{ABSTRACT}

The SOFIE (Intelligent Assisted Bicycles) project wishes to create performance and design guidelines for mechatronic appliances which improve the stability of electric bicycles, so-called intelligent stability assist devices (IAD). To achieve this goal, a stability hypothesis, an advanced rider/bicycle model and bicycle stability test bench, will be created. This paper describes the development of these components and its goal is to present the project design. The stability hypothesis is based on the concept that the Centre of Mass (CoM) of the bicycle/rider system stays within certain lateral margins from the heading of a bicycle. The rider/bicycle model is created in Adams for multi-body dynamic simulations. The bicycle stability test bench is designed to be interchangeable between bicycles. The model, the test bench and the stability hypothesis will be used to validate the effectiveness of the IAD's and assist in their design.

\section{NOMENCLATURE}

SOFIE Slimme Ondersteunde FIEts (Dutch). Intelligent assisted bicycles.

IAD Intelligent Assist Device.

IMU Inertial Measurement Unit.

CoM Centre of Mass.

CoP Center of Pressure.

\section{INTRODUCTION}

Bicycle dynamics and stability has been a subject of interest for many years [1,2]. Studying the stability of the bicycle can lead to design guidelines for more stable bicycles. Within the SOFIE-project we wish to add mechatronic appliances to electric bicycles in order to improve the bicycle/rider system stability and thereby increase the safety of cycling.

Rider movements and rider perception are important when assessing the stability of a bicycle rider. The rider needs to feel comfortable and confident. Most studies focus only on the stability of the vehicle and ignore the influence of the rider [3, 4]. Their models use linearisation techniques and a simplified tyreroad interaction model, which could lead to overlooking the real problem. To realistically assess stability of a bicycle-rider system a more detailed system description is necessary.

In order to create performance and design guidelines for the Intelligent Assist Devices (IAD), methods and tools to assess stability need to be developed. A detailed model of the bicycle-rider system as well as a test bench which is able to measure all the important parameters for stability are necessary. The model and test bench are tightly connected and dependent on each other. A stability criterion determines whether the system is in a stable situation. Such a criterion does not yet exist in literature for cycling.

In this paper we present a multi-body model, a test bench and a new stability hypothesis for cycling based on criteria pre- 


\section{Stability Margin Definitions}

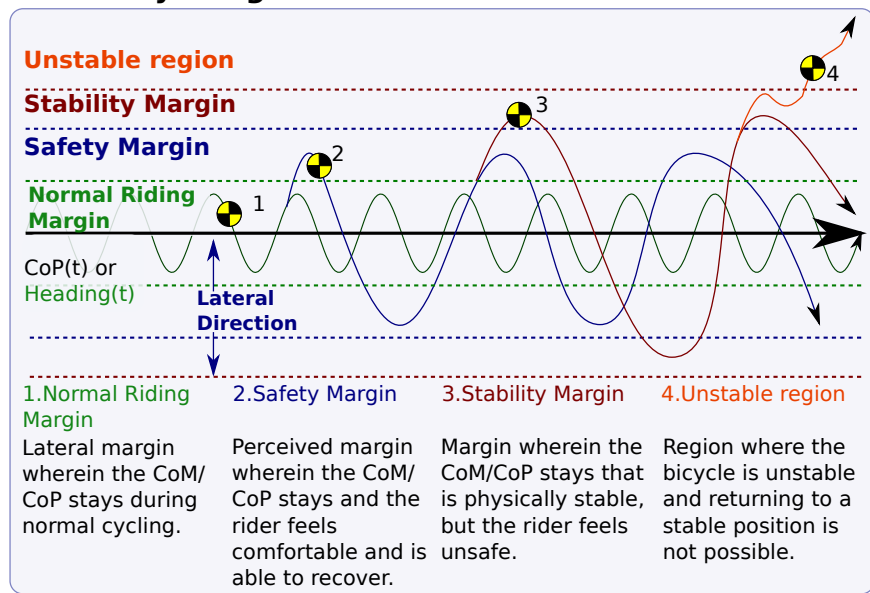

\section{Hypothesis Definitions 3.Margin calculation}

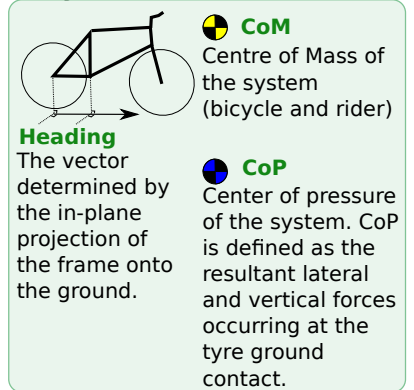

Com and CoP
are 'near' to
the same line
between the
two contact
points (which
is not
necessarily
on the
heading).
Scenarios to the
line between
the two contact
points.
Straight line
cycling.
Scenarios
Steady state
corner.

* Top view of bicycle with the two wheel contacts.
4.Cycling Scenarios*

\begin{abstract}
Margin dependent on the rider, forward velocity and cycling scenario.

Stability hypothesis version 1 The maximum difference between the CoM and the Heading in lateral direction determines the margins.

Stability hypothesis version 2 The maximum difference between the $\mathrm{CoP}$ and the Heading in lateral direction determines the margins.

Stability hypothesis version 3 The maximum difference between the CoM and the CoP determines the margins, with the CoP as the reference.
\end{abstract}

5. Required Parameters

Bicycle CoM Bicycle CoP Bicycle 3-D dimensions, accelerations of lean and the bicycle and steering angle. tyre-road Rider CoM Dimension of Rider CoP torso, legs, Torso, legs, head, arms and head, arms head, arms and head, arms
their movements. Bicycle lean angle and frame dimensions.

6. Experiments

Normal Riding Stability Margin Margin model lab/outdoor (lab/outdoor). Safety Margin Unstable region lab/outdoor subjective (human) (lab/outdoor). measurements.

Figure 1. THE SOFIE STABILITY HYPOTHESIS. lateral margins from the heading of a bicycle and is derived from definitions of stability used in stance and gait analysis. This hypothesis will now be discussed by making use of the Fig. 1 as the main resource for its description.

The definition of the CoM, CoP and Heading is described in Block 2 in Fig. 1. The hypothesis defines a number of stability margins in Block 1 in Fig. 1. The first version of the hypothesis will use the CoM for the margin calculations and if needed further developed to use the CoP (see Block 3 in Fig. 1) for a more complex/accurate stability hypothesis.

The objective stability margins (Normal Riding Margin and Stability Margin) are functions of the rider who is cycling, the velocity and cycling scenario (see Block 4 in Fig. 1 for cycling scenario descriptions.) The subjective margin, i.e. the Safety Margin, will be determined from the perceived feeling of safety of the rider. The Stability Margin will possibly be determined from experiments but most likely determined using the model because of safety concerns.

This design of the stability hypothesis is able to measure the physical stability of the rider/bicycle system and the riders perceived safety. The combined result leads to a method that is able to analyse the benefit of an IAD to the physical system and to the riders feeling of safety.

\section{Stability hypothesis verification}

Existence of the Safety margin in the order shown in Block 1 in Fig. 1 is based on the assumption that people experience these different regions of stability. After the performance of experiments it could turn out that this order is not correct. For example, in more challenging cycling tasks the normal riding margin may expand and 'touch' the safety margin. The Safety Margin, will be determined from the riders feedback: A questionnaire will include individual cycling task scoring regarding the feelings of experienced safety and fear of falling.

Block number 5 in Fig. 11 describes what is required for the estimation and calculation of the CoM, CoP and Heading. Block 6 in Fig. 1 gives a brief description of experiments that are needed to determine the different margins. The model will be used to aid in the calculation of the CoM and CoP. The bicycle stability test bench section will describe how the different parameters required to prove the SOFIE stability hypothesis will be measured.

\section{MODEL}

An advanced multi-body model will be used to assess the stability of a bicycle-rider system in several problem scenario's (such as riding at low velocity, cornering at high velocities and during sudden avoidance's/perturbations) and help in the calculation of the CoM and CoP. The model should be able to simulate any kind of bicycle and/or rider (using a parametrised model) within the target group. The model will be used to gain insight into the effect of different parameters on the stability of the system, which could lead to design guidelines for the IAD's. Fur-

\section{SOFIE STABILITY HYPOTHESIS}

The SOFIE project proposes a working hypothesis based on subjective and objective measurements to qualify and quantify cycling stability respectively. The subjective stability parameter refers to the feeling of stability of the cyclist. The objective stability parameter is based on the concept that the Centre of Mass (CoM) of the bicycle/rider system stays within certain 


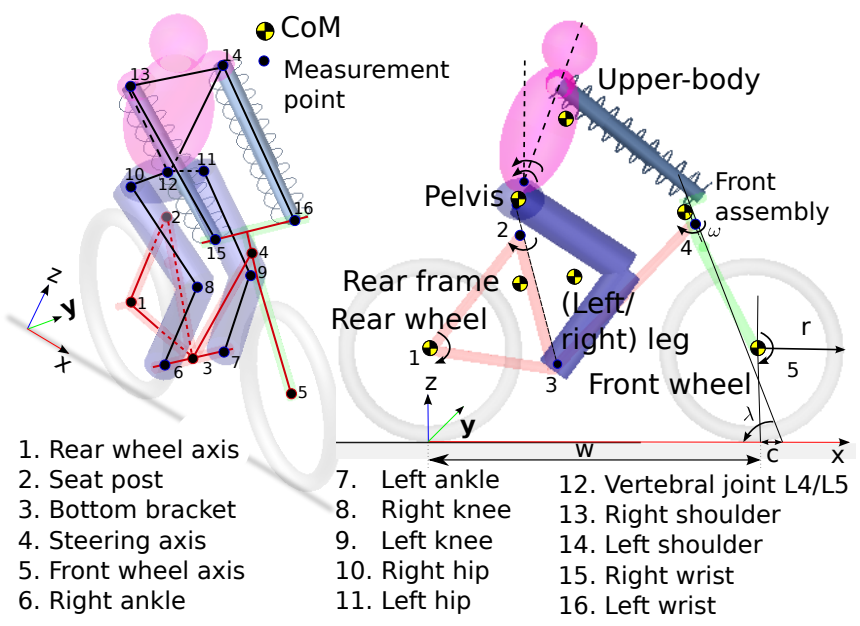

Figure 2. BICYCLE AND RIDER MODEL

thermore the model will be used to test preliminary IAD concepts and used along with the test bench and stability hypothesis to evaluate the performance of the IAD's.

Literature review has revealed some limitations of existing bicycle/(rider) models: a simplified tyre-road contact model, restrictions to only small deviations from an equilibrium configuration, lack of a good rider model (no mechanical behavioural response), lack of realistic control behavioural response of the rider, lack of a stability criterion $[4,7,8,9,10,11]$. Cain et al. found that a simple model is does not predict the steering torque well, especially when the rider leans. This could be caused by simplifications in the model [12]. Motorcycle dynamic models already include some of these features [13], for example Cossalter et al. developed a passive rider model and a 3D road-tire model [14].

A new 3D model is presented here, which combines the bicycle dynamics, an advanced tyre-road contact model, the biomechanics of the rider, its mechanical and control responses and interactions with the environment. The model is constructed with the software package Adams (http://www.mscsoftware. com/adams) for multi-body dynamic simulations.

\section{Bicycle and Rider Dynamics}

The bicycle dynamics are captured in four rigid bodies: the rear frame, front-assembly, rear wheel and front wheel as depicted in Fig. 2. The wheels are interconnected with the frame by revolute joints. The rear frame and front assembly are connected by a revolute joint at the steering axis. A torque around the steering axis simulates steering inputs and an actuation torque on the rear wheel axis regulates the forward speed.

Another four rigid bodies represent the dynamics of the rider: the upper-body (torso, head and upper arms), the pelvis and the two legs. The pelvis is fixed to the frame of the bicycle. Upper-body movement is accomplished by a spherical joint between the pelvis and the upper-body at the position of the vertebral joint L4/L5, allowing rotation in all three directions. The

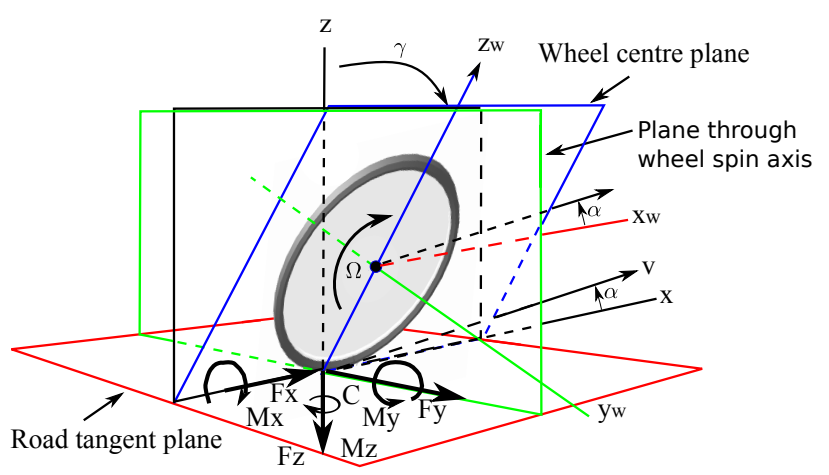

Figure 3. DEFINITIONS OF THE TYRE MODEL ACCORDING TO SAE STANDARD: $\mathrm{x}, \mathrm{y}, \mathrm{z}$ represent the global coordinate system, $x_{w}, y_{w}$ and $z_{w}$ represent the coordinate system in the wheel centre. $\mathrm{C}=$ contact point, $v$ =forward velocity, $F_{z}=$ vertical force, $F_{x}=$ longitudinal force, $F_{y}=$ lateral force, $M_{x}=$ overturning moment, $M_{y}=$ rolling resistance moment, $M_{z}=$ yawing moment, $\alpha=$ side-slip angle, $\gamma=$ camber angle and $\Omega$ the angular velocity (Image derived from [18]).

legs have one degree of freedom: rotation around an axis through the hip and ankle, enabling lateral knee movements. Joint stiffnesses are modelled using a torsion spring/damper around the rotation axis of the joint, to prevent uncontrolled movement of rider parts. The torso is connected to the handlebars by two spring/dampers which allow steering movements. The stiffness of the springs controls the tightness of the riders grip (cocontraction of arm muscles) on the handlebars.

Preliminary geometry and mass properties of the bicycle are based on an experimental bicycle (representative for a typical Dutch electric bicycle), which is used for validation experiments. The rider dimensions and mass properties were based on an average person [15]. Figure 2 shows the 16 measured points from which the model is built. The model will be parametrised.

A control model will be developed based on human balance control models used in stance and gait [16]. Output of this model are steering torques and torques which actuates lateral knee movement and upper-body lean (the three control mechanisms used by the rider to control the bicycle [17]).

\section{Tyre-Road Contact Model}

The tyre-road contact is modelled with the 'Pacejka 2002' tyre-road contact model available in Adams/tyre [19]. The models developed by Pacejka were named the 'Magic Formula', because they have no particular physical meaning, but can be used to fit a wide variety of tyre behaviour [19,20]. Each tyre is characterised by 10-99 coefficients in total, fitting experimental data to the model. The general form of the Magic Formula is shown in the equation below, were $b, c, d$ and $e$ represent fitting coefficients and $R$ a force or moment resulting from a slip parameter $k$.

$$
R(k)=d \cdot \sin (c \cdot \arctan (b(1-e) k+e \cdot \arctan (b k)))
$$




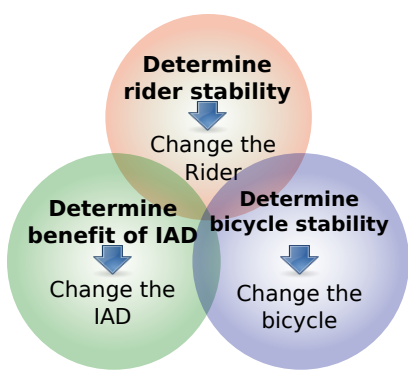

Figure 4. THE BICYCLE STABILITY TEST BENCH GOALS.

Experimental data measured with the rotating disc machine, found at the University of Padova, is used to derive the Magic formula coefficients and these results will be published elsewhere. Input to the model are tyre and road data (road data contains dimensions, shape and a value for the road friction $(\mu)$, tyre data includes tyre dimensions, mass properties, stiffness and damping values and the Magic Formula coefficients). From this input the load and slip of the wheel are calculated, output are the forces $\left(F_{z}, F_{x}, F_{y}\right)$ and the moments $\left(M_{z}, M_{x}, M_{y}\right)$ at the contact point $C$ (defined as the intersection of the wheel centre plane with the road tangent plane and the plane trough the wheel spin axis, as depicted in Fig. 37. In the radial direction this model considers the tyre to behave as a parallel linear spring/damper. The lateral force depends on the side-slip angle $\alpha$ and the camber angle $\gamma$ (see Fig. 3 for the definitions). The tyre deforms with the contact on the ground and the contact patch (the portion of the tyre which is in actual contact with the road surface) variates with characteristics of the tyre, the camber angle, the side-slip angle and external factors, such as the load and inflation pressure.

\section{BICYCLE STABILITY TEST BENCH}

The goals and requirements of the bicycle stability test bench are shown in Fig. 4. Thus the test bench is designed in a manner whereby it is easily interchangeable between bicycles for many different types of bicycles.

The test bench will be used for a number of different functions within the project. The model needs to be validated, thus we need to have data to validate against. These data will be created by the test bench. The stability hypothesis needs to be proven or disproved, thus experiments are performed to achieve this.

\section{Design}

The test bench is designed with an architecture to enable it to fulfil the goals of the test bench, model validation and stability hypothesis verification and adapt to changing insights and requirements as the project progresses. The design has five different sub-systems each shown in its own coloured block in Fig. 5 and described below:

1. A 'Data processing back-bone' is designed to standardise

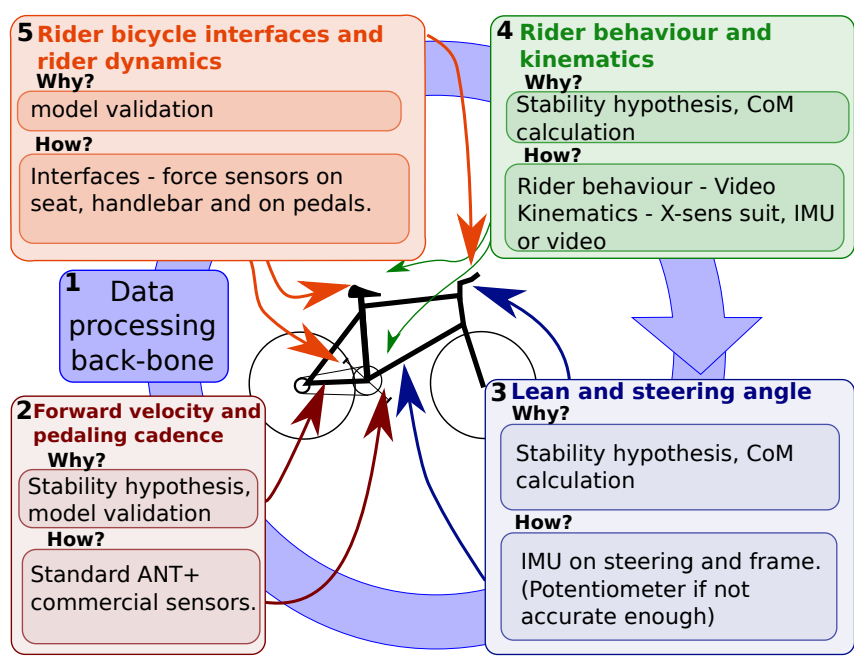

Figure 5. THE BICYCLE STABILITY TEST BENCH DESIGN

the processing of data. The 'SOFIE-HDF-FORMAT' (https://github.com/agcooke/Sofie-HDF-Format) software tool was created to achieve this. It handles the conversion of data from different systems to a common format which is easily processed by the various software tools in the project (Python, Matlab and Adams).

2. The 'Forward velocity and cadence' subsystem is created using commercial ANT+ sensors. ANT+ (http://www. thisisant.com) is an open wireless communication protocol used in the fitness industry to communicate between lowpower sensor devices and a central data-processing unit. Additional ANT+ sensors (such as pedalling power sensors) can be added at a later stage.

3. The 'Lean and steering angle' sub-system is being designed to use a modern inertial measurement unit (IMU) from Inertia Technology (http://inertia-technology.com/). The difference between the IMU measured angles on the bicycle frame and on the steering column will be used to determine the steering angle.

4. The 'Rider behaviour and kinematics' will include a video camera system. The video camera's will be used to evaluate an experiment if we observe anomalies in the data and to interpret rider behaviour. The rider kinematics will be measured using an X-sens MVN (http://www.Xsens.com/) suit, VICON markers and eventually a simplified system using video or a minimal number of IMU's.

5. The 'Rider bicycle interfaces and rider dynamics' are needed to validate the model and determine how a rider controls the bicycle. We are going to create force sensors on the handlebars (where the rider grips the handlebar), on the pedals using an off the shelf fitness product and on the seat. This sub-system is still in the conceptual design phase.

Blocks 1 to 3 in Fig. 5 have been implemented and preliminary data are measured. 


\section{DISCUSSION AND FUTURE WORK}

The incorporation of objective and subjective criteria in the SOFIE stability hypothesis creates a comprehensive measure of stability, in real world scenario's. This will create a novel stability performance measure which will aid in the design of not only dynamically stable bicycles and IAD's, but also bicycles that the cyclist perceives as safer.

The presented advanced multi-body model is the first model which combines bicycle dynamics, rider bio-mechanics, rider control and an advanced tyre-road contact model. It is able to simulate behaviour in the non-linear regime, unlike most other models. This is required because bicycle accidents occur during situations which are highly non-linear, like manoeuvring due to slippery roads. A disadvantage of the model is the complexity, which complicates the isolation of the effect of a single parameter. Therefore an extensive sensitivity study and parameter analysis is required.

The design of the test bench will enable it to be used in versatile situations, a possible side-effect of this versatility is the increase in complexity required to synchronise and analyse data.

\section{Future work}

The test bench needs to be used to validate the model against the benchmark model [4] to prove that the model is a valid representation of the dynamics of riding a bicycle. The model will be parametrised to be able to model any type of bicycle and rider. Furthermore the model will need to design and implement a rider control model.

Once the SOFIE project has a validated model and functional test bench system, the robustness of the SOFIE stability hypothesis needs to be thoroughly investigated. A simulator environment is a possible further development to a more advanced experimentation set-up for bicycle research.

\section{REFERENCES}

[1] Whipple, F. J. W., 1899. "The stability of the motion of a bicycle". Quarterly Journal of Pure and Applied Mathematics, 30, pp. 312-348.

[2] Schwab, A. L., Kooijman, J. D. G., and Meijaard, J. P., 2008. "Some recent developments in bicycle dynamics and control". In Fourth European Conference on Structural Control (4ECSC), A. K. Belyaev and D. A. Indeitsev, eds., Institute of Problems in Mechanical Engineering, Russian Academy of Sciences, pp. 695-702.

[3] Chen, C.-K., and Dao, T.-S., 2007. "Genetic fuzzy control for path-tracking of an autonomous robotic bicycle". Journal of System Design and Dynamics, 1, pp. 536-547.

[4] Schwab, A. L., Meijaard, J. P., and Papadopoulos, J. M., 2005. "A multibody dynamics benchmark on the equations of motion of an uncontrolled bicycle". In ENOC, Vol. 19, The Korean Society of Mechanical Engineers, pp. 292-304.

[5] Hof, A. L., Gazendam, M. G., and Sinke, W. E., 2005. "The condition for dynamic stability.”. J Biomech, 38(1), Jan., pp. 1-8.

[6] Pratt, J. E., and Tedrake, R., 2006. "Velocity-based stability margins for fast bipedal walking". pp. 299-324.

[7] Schwab, A. L., Meijaard, J. P., and Kooijman, 2011. "Lateral dynamics of a bicycle with a passive rider model: stability and controllability". Vehicle System Dynamics, 0(0), pp. 1-16.

[8] Hess, R., Moore, J. K., and Hubbard, M., 2012. "Modeling the manually controlled bicycle". Systems, Man and Cybernetics, Part A: Systems and Humans, IEEE Transactions on, PP(99), pp. 1-13.

[9] Papadopoulos, J. M., 2010. "Critique of assumptions underlying bicycle handling research". In Bicycle and Motorcycle Dynamics. Symposium on the Dynamics and Control of Single Track Vehicles, TU Delft, the Netherlands.

[10] Cangley, P., Passfield, L., Carter, H., and Bailey, M., 2011. "A model for performance enhancement in competitive cycling". Movement \& Sport Sciences - Science \& Motricité(75), Oct., pp. 59-71.

[11] Dressel, A., and Rahman, A., 2011. "Measuring sideslip and camber characteristics of bicycle tyres". Vehicle System Dynamics, 0(0), pp. 1-14.

[12] Cain, S. M., and Perkins, N. C., 2012. "Comparison of experimental data to a model for bicycle steady-state turning". Vehicle System Dynamics, 0(0), pp. 1-24.

[13] Sharp, R., Evangelou, S., and Limebeer, D., 2004. "Advances in the modelling of motorcycle dynamics". Multibody System Dynamics, 12, pp. 251-283. 10.1023/B:MUBO.0000049195.60868.a2.

[14] Cossalter, V., Lot, R., and Massaro, M., 2011. “An advanced multibody code for handling and stability analysis of motorcycles". Meccanica, 46, pp. 943-958. 10.1007/s11012-010-9351-7.

[15] Winter, D., 1990. Biomechanics and Motor COntrol of Human Movement. BIOMEDICAL ENGINEERING and HEALTH SYSTEMS: a WILEY-INTERSCIENCE SERIES Series. Wiley.

[16] Peterka, R. J., 2003. "Simplifying the complexities of maintaining balance". Engineering in Medicine and Biology Magazine, IEEE, 22(2), Mar., pp. 63-68.

[17] Moore, J. K., Kooijman, J. D. G., and Schwab, A. L., 2009. "Rider motion identification during normal bicycling by means of principal component analysis". In Multibody Dynamics 2009, ECCOMAS Thematic Conference, K. Arczewski and Frcaczek, eds.

[18] Msc SOFTwARE, 2005. PAC MC ADAMS/Tire 2005 r2 Help.

[19] Pacejka, H. B., 2005. Tire and Vehicle Dynamics, 1 ed. SAE International, Dec.

[20] Lot, R., 2004. "A motorcycle tire model for dynamic simulations: Theoretical and experimental aspects". Meccanica, 39(3), June, pp. 207-220. 